\title{
The birth of monoclonal antibodies
}

On 7 August 1975, Nature published a threepage report by César Milstein and Georges J. F. Köhler describing a method for generating large amounts of monoclonal antibodies of a predefined specificity. Not only did this method revolutionize biomedical research and diagnostics (MILESTONE 4), it also led to the generation of an arsenal of therapies for many diseases (MILESTONES 13, 14).

The story of monoclonal antibodies began with the arrival of the Argentinean émigré César Milstein at the Medical Research Council Laboratory of Molecular Biology, Cambridge, UK, in 1963. Encouraged by Fred Sanger, who at the time was head of the Protein and Nucleic Acid Chemistry Division at the Laboratory of Molecular Biology, Milstein began investigating how antibody diversity is generated (MILESTONE 10). A key issue that was restricting the whole field at the time was the inability to isolate and purify single antibodies of known specificity from the billions produced in the body.

Henry Kunkel, based at the Rockefeller Institute in New York, had noted in 1951 that myeloma cells produce antibodies of just a single specificity; therefore, myeloma cell lines had become an important tool in antibody research. Dick Cotton, an Australian postdoctoral scientist new to the Milstein laboratory, showed in 1973 that the fusion of two immunoglobulin-producing myeloma cell lines produced a hybrid cell line that secreted myeloma antibodies of both parental types. Although the specificity of the antibodies produced was not known, this study was important for future work, as it described a technique for the generation of hybrid clones.

While presenting that work at the Basel Institute of Immunology, Milstein met the German Ph.D. student Georges Köhler, who joined Milstein's laboratory as a postgraduate in April 1974. Although several methods for cloning B cells that produce single antibodies of known specificity had been described-in particular, a technique developed by Norman Klinman at the University of Pennsylvania-these approaches had several limitations, including low antibody yield and short cell lifespan. Köhler and Milstein's solution was to generate a hybridoma. With the help of the laboratory technician Shirley Howe, they fused cells from the myeloma cell line P3-X63-Ag8 (which Cotton had been

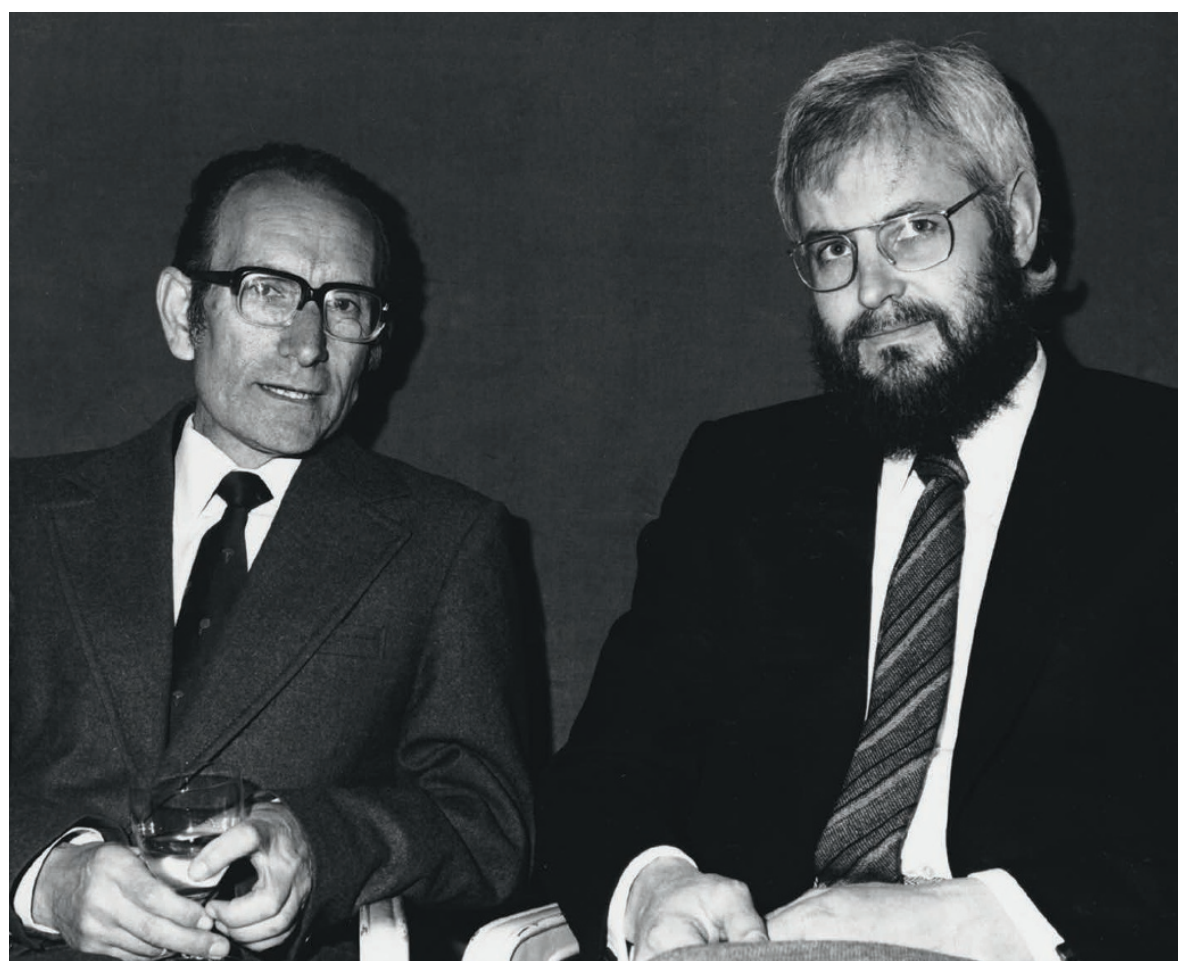

César Milstein and Georges Köhler together in 1984, the year they were awarded the Nobel Prize in physiology or medicine, jointly with Niels Jerne. Photo reproduced courtesy of the Celia Milstein and the MRC Laboratory of Molecular Biology, Cambridge, UK.

working on) with spleen cells from a mouse immunized with sheep red blood cells. The cells were then incubated in hypoxanthine aminopterin thymidine medium for up to 2 weeks; myeloma cells lack expression of the enzyme hypoxanthine guanine phosphoribosyltransferase, which is necessary for cell growth in this medium, and unfused $\mathrm{B}$ cells have a short lifespan. Therefore, only the fused cells survive. In this way they generated a hybridoma with the immortality of the myeloma cell and the ability to produce antibodies specific for sheep red blood cells, which originated from the spleen cell. After overcoming some technical difficulties, they successfully generated several hybridomas that secreted very large quantities of monoclonal antibodies.

In the Nature paper describing their discovery, Köhler and Milstein hinted at the potential implications of their findings, stating that "[s]uch cultures could be valuable for medical and industrial use." However, the importance of the paper was largely overlooked at the journal, and it was omitted from the section reserved for findings of leading importance. It took a couple of years for the broader implications of the discovery to be realized, but with the realization of the potential advantages of the technique, as outlined in an editorial in the Lancet in June 1977 claiming the discovery could "have profound implications for medical practice", Milstein soon became inundated with requests for cell samples. This paved the way for the early commercialization of monoclonal antibody technology.

The contributions made by Köhler and Milstein to scientific research were formally recognized when they were presented with both the Albert Lasker Basic Medical Research Award and the Nobel Prize in physiology or medicine, jointly with Niels Kaj Jerne, in 1984.

Olive Leavy,

Chief Editor, Nature Reviews Immunology

ORIGINAL RESEARCH PAPERS Cotton, R. G. H. \& Milstein, C. Fusion of two immunoglobulin-producing myeloma cells. Nature 244, 42-43 (1973) | Klinman, N. R. Antibody with homogeneous antigen binding produced by splenic foci in organ culture. Immunochemistry 6, 757-759 (1969) | Köhler, G. \& Milstein, C. Continuous cultures of fused cells secreting antibody of predefined specificity. Nature 256, 495-497 (1975) FURTHER READING http://www.whatisbiotechnology.org/ exhibitions/milstein/antibodies 